Commun. Korean Math. Soc. 29 (2014), No. 1, pp. 123-130

http://dx.doi.org/10.4134/CKMS.2014.29.1.123

\title{
STABLE MINIMAL HYPERSURFACES WITH WEIGHTED POINCARÉ INEQUALITY IN A RIEMANNIAN MANIFOLD
}

\author{
Nguyen Dinh Sang and Nguyen Thi Thanh
}

\begin{abstract}
In this note, we investigate stable minimal hypersurfaces with weighted Poincaré inequality. We show that we still get the vanishing property without assuming that the hypersurfaces is non-totally geodesic. This generalizes a result in [2].
\end{abstract}

\section{Introduction}

Let $M$ be a complete noncompact Riemannian manifold. Let $\lambda_{1}(M)>0$ denote the lower bound of the spectrum of the Laplacian on $M$, namely,

$$
\lambda_{1}(M):=\inf \left\{\frac{\int_{M}|\nabla \phi|^{2}}{\int_{M} \phi^{2}}: \phi \in \mathcal{C}_{0}^{\infty}(M)\right\} .
$$

Lam proved the following vanishing type theorem.

Theorem 1.1 (Lam, [3]). Let $M$ be a complete non-compact Riemannian manifold and $h$ be a function which is defined on $M$ satisfying

$$
h \Delta h \geq-a h^{2}+b|\nabla h|^{2} .
$$

Assume that $\lambda_{1}(M)>0$ and the Ricci curvature of $M$ satisfies

$$
\operatorname{Ric}_{M} \geq-(b+1) \lambda_{1}(M)+\delta
$$

for some $\delta>0$. If $\int_{B_{p}(R)} h^{2}=o\left(R^{2}\right)$, then $h \equiv 0$.

Lam's theorem implies the following result which was proved by Li-Wang $([5])$.

Theorem 1.2 (Li-Wang, [5], Theorem 4.2). Let $M^{n}$ be a complete non-compact $n$-dimensional Riemannian manifold with $\lambda_{1}(M)>0$ and the Ricci curvature satisfies

$$
\operatorname{Ric}_{M} \geq-\frac{n}{n-1} \lambda_{1}(M)+\delta
$$

Received September 10, 2012.

2010 Mathematics Subject Classification. Primary 53C42, 58C40.

Key words and phrases. minimal hypersurface, stability, weighted Poincaré inequality. 
for some $\delta>0$. Then

$$
H^{1}\left(L^{2}(M)\right):=\left\{\omega \text { is a harmonic 1-form, } \int_{M}|\omega|^{2}<+\infty\right\}=0 .
$$

In the other direction, Dung and Seo ([2]) considered a complete stable minimal hypersurface in a Riemannian manifold with sectional curvature bounded below by a nonpositive constant and proved the following theorem.

Theorem 1.3. Let $N$ be $(n+1)$-dimensional Riemannian manifold with sectional curvature $K_{N}$ satisfying $K \leq K_{N}$ where $K \leq 0$ is a constant. Let $M$ be a complete noncompact stable non-totally geodesic minimal hypersurface in $N$. Assume that

$$
-K(2 n-1)(n-1)<\lambda_{1}(M) .
$$

Then there are no nontrivial $L^{2}$ harmonic 1-forms on $M$.

The main purpose of this note is to study structure theorems for submanifolds satisfying a weighted Poincaré type property. The submanifold satisfying a weighted Poincaré inequality is studied in [1], recently. First, let us first recall some definitions.

Definition. Let $M^{m}$ be an $m$-dimensional complete Riemannian manifold. We say that $M^{m}$ has property $\left(\mathcal{P}_{\rho}\right)$ if a weighted Poincaré inequality is valid on $M$ with some nonnegative weight function $\rho$, namely

$$
\int_{M} \rho(x) \phi^{2} \leq \int_{M}|\nabla \phi|^{2}
$$

is valid for all compactly supported smooth functions $\phi \in C_{0}^{\infty}(M)$. Moreover, the $\rho$-metric, defined by

is complete.

$$
d s_{\rho}^{2}=\rho d s_{M}^{2}
$$

In particular, if $\lambda_{1}(M)$ is assumed to be positive, then obviously $M$ possesses property $\left(\mathcal{P}_{\rho}\right)$ with $\rho(x)=\lambda_{1}(M)$. The notion of property $\left(\mathcal{P}_{\rho}\right)$ may be viewed as a generalization of the assumption $\lambda_{1}(M)>0$.

In this paper, motivated by all the above results, we prove the following main theorem.

Theorem 1.4. Let $N$ be $(n+1)$-dimensional Riemannian manifold, and $M$ be a complete noncompact stable minimal hypersurface in $N$ with $\left(\mathcal{P}_{\rho}\right)$ property for some non-negative weight function $\rho$ defined on $M$. Assume that

$$
K_{N}(x) \geq-\frac{(1-\delta) \rho(x)}{(2 n-1)(n-1)} \text { for all } x \in M
$$

for some $\delta>0$, and $K_{N}$ stands for sectional curvature of $N$. If $\rho=o\left(r^{2-\alpha}\right)$ for some $0<\alpha<2$, then there are no nontrivial $L^{2}$ harmonic 1-forms on $M$. 
Note that we do not require $M$ is non-totally geodesic. Moreover, we only need to have the lower bound of $K_{N}$ on $M$. Hence, this result is a generalization and refinement of Theorem 1.3.

Corollary 1.5. Let $N$ be $(n+1)$-dimensional Riemannian manifold, and $M$ be a complete noncompact stable minimal hypersurface in $N$ with $\lambda_{1}(M)>0$. Assume that

$$
K_{N} \geq-\frac{\lambda_{1}(M)}{(2 n-1)(n-1)}+\delta
$$

for some $\delta>0$, and $K_{N}$ stands for sectional curvature of $N$. Then there are no nontrivial $L^{2}$ harmonic 1-forms on $M$.

This note is divided into two sections. Beside this section, in Section 2, we prove the our main theorem and give an application to study a geometric property of submanifolds.

\section{Vanishing theorem on minimal hypersurfaces}

We first recall some useful results which we shall use in this section. The following lemma holds true on any complete manifold $M$.

Lemma 2.1 ([3]). Let $M$ be a complete manifold and $b>-1$. Assume that $h$ is a nonnegative function on $M$ and satisfies the differential inequality $h \Delta h \geq$ $-a h^{2}+b|\nabla h|^{2}$ in the weak sense, for some function $a>0$. For any $\varepsilon>0$, we have the following estimate

$$
(b(1-\varepsilon)+1) \int_{M}|\nabla(\phi h)|^{2} \leq\left\{b\left(\frac{1}{\varepsilon}-1\right)+1\right\} \int_{M} h^{2}|\nabla \phi|^{2}+\int_{M} a \phi^{2} h^{2}
$$

for any compactly supported smooth function $\phi \in \mathcal{C}_{0}^{\infty}(M)$. In addition, if

$$
\int_{B_{p}(R)} h^{2}=o\left(R^{2}\right),
$$

then

$$
\int_{M}|\nabla h|^{2} \leq \frac{1}{b+1} \int_{M} a h^{2}
$$

In particular, if a is bounded, then $h$ has finite Dirichlet integral if $h \in L^{2}(M)$.

Leung (see [4]) proved the following estimate on the Ricci curvature of a submanifold.

Lemma $2.2([4])$. Let $M$ be an $n$-dimensional complete immersed minimal hypersurface in a Riemannian manifold $N$. If all the sectional curvatures of $N$ are bounded pointwise from below by a function $K$, then

$$
R i c \geq(n-1) K-\frac{n-1}{n}|A|^{2} .
$$


We should note in [4], the author assumed that all the sectional curvatures of $N$ are bounded below by a constant $k$. But according to his argument, this assumption was only used in the end of the proof, hence his method can be used to prove the above lemma without any change. For harmonic 1-forms, one has the Kato-type inequality as follows:

Lemma 2.3 ([6]). Let $\omega$ be a harmonic 1 -form on an $n$-dimensional Riemannian manifold $M$. Then

$$
|\nabla \omega|^{2}-|\nabla| \omega||^{2} \geq \frac{1}{n-1}|\nabla| \omega||^{2} .
$$

We shall generalize Theorem 1.3 to a complete stable minimal hypersurface with $\left(\mathcal{P}_{\rho}\right)$ property in a Riemannian manifold in which sectional curvature bounded below by a nonpositive function as follows.

Theorem 2.4. Let $N$ be $(n+1)$-dimensional Riemannian manifold, and $M$ be a complete noncompact stable minimal hypersurface in $N$ with $\left(\mathcal{P}_{\rho}\right)$ for some nonnegative weight function $\rho$. Assume that

$$
K_{N} \geq-\frac{(1-\delta) \rho}{(2 n-1)(n-1)}
$$

for some $\delta>0$, and $K_{N}$ stands for sectional curvature of $N$. If $\rho=o\left(r^{2-\alpha}\right)$ for some $0<\alpha<2$, then there are no nontrivial $L^{2}$ harmonic 1 -forms on $M$.

Proof. Let $\omega$ be an $L^{2}$ harmonic 1-form on M, i.e.,

$$
\Delta \omega=0 \text { and } \int_{M}|\omega|^{2} d v<\infty .
$$

In an abuse of notation, we will refer to a harmonic 1-form and its dual harmonic vector field both by $\omega$. From Bochner formula, it follows

$$
\Delta|\omega|^{2}=2\left(|\nabla \omega|^{2}+\operatorname{Ric}(\omega, \omega)\right) .
$$

On the other hand, one sees that

$$
\Delta|\omega|^{2}=2\left(|\omega| \Delta|\omega|+|\nabla| \omega||^{2}\right) .
$$

Thus we obtain

$$
|\omega| \Delta|\omega|-\operatorname{Ric}(\omega, \omega)=|\nabla \omega|^{2}-|\nabla| \omega||^{2} .
$$

Applying Lemma 2.2 and Kato-type inequality (1) yields

$$
|\omega| \Delta|\omega|+\frac{n-1}{n}|A|^{2}|\omega|^{2}+\frac{(1-\delta) \rho}{2 n-1}|\omega|^{2} \geq\left.\frac{1}{n-1}|\nabla| \omega\right|^{2} .
$$

The stability of $M$ implies that

$$
\int_{M}\left(|\nabla \phi|^{2}-\left(|A|^{2}+\overline{\operatorname{Ric}}\left(e_{n+1}\right)\right) \phi^{2}\right) \geq 0
$$


for any compactly supported Lipschitz function $\phi$ on $M$. Since

$$
-\frac{n(1-\delta) \rho}{(n-1)(2 n-1)} \leq \overline{\operatorname{Ric}}\left(e_{n+1}\right)
$$

we have

Replacing $\phi$ by $|\omega| \phi$ gives

$$
\int_{M}\left(|\nabla \phi|^{2}-\left(|A|^{2}-\frac{n(1-\delta) \rho}{(n-1)(2 n-1)}\right) \phi^{2}\right) \geq 0 .
$$

$$
\int_{M}\left(|\nabla(|\omega| \phi)|^{2}-\left(|A|^{2}-\frac{n(1-\delta) \rho}{(n-1)(2 n-1)}\right)(|\omega| \phi)^{2}\right) \geq 0 .
$$

Applying the divergence theorem, we get

$$
\begin{aligned}
0 \leq & -\int_{M}|\omega| \phi \Delta(|\omega| \phi)-\int_{M}\left(|A|^{2}-\frac{n(1-\delta) \rho}{(n-1)(2 n-1)}\right)|\omega|^{2} \phi^{2} \\
= & -\int_{M} \phi|\omega|^{2} \Delta \phi-\int_{M} \phi^{2}\left(|\omega| \Delta|\omega|+|A|^{2}|\omega|^{2}\right)-2 \int_{M} \phi|\omega|\langle\nabla|\omega|, \nabla \phi\rangle \\
& +\frac{n(1-\delta)}{(n-1)(2 n-1)} \int_{M} \rho \phi^{2}|\omega|^{2} \\
= & \int_{M}\left\langle\nabla\left(\phi|\omega|^{2}\right), \nabla \phi\right\rangle-\int_{M} \phi^{2}\left(|\omega| \Delta|\omega|+|A|^{2}|\omega|^{2}\right)-2 \int_{M} \phi|\omega|\langle\nabla|\omega|, \nabla \phi\rangle \\
& +\frac{n(1-\delta)}{(n-1)(2 n-1)} \int_{M} \rho \phi^{2}|\omega|^{2} \\
= & \int_{M}|\omega|^{2}|\nabla \phi|^{2}-\int_{M} \phi^{2}\left(|\omega| \Delta|\omega|+|A|^{2}|\omega|^{2}\right)+\frac{n(1-\delta)}{(n-1)(2 n-1)} \int_{M} \rho \phi^{2}|\omega|^{2} .
\end{aligned}
$$

Using the inequalities (2) and (3), we obtain

$$
0 \leq \int_{M}|\omega|^{2}|\nabla \phi|^{2}+\frac{(1-\delta)}{n-1} \int_{M} \rho \phi^{2}|\omega|^{2}-\frac{1}{n-1} \int_{M} \phi^{2}|\nabla| \omega||^{2}-\frac{1}{n} \int_{M}|A|^{2} \phi^{2}|\omega|^{2} .
$$

From the assumption on weighted Poincaré inequality, it follows

$$
\begin{aligned}
\int_{M} \rho \phi^{2}|\omega|^{2} & \leq \int_{M}|\nabla(\phi|\omega|)|^{2} \\
& =\int_{M}|\omega|^{2}|\nabla \phi|^{2}+\left.\phi^{2}|\nabla| \omega\right|^{2}+2|\omega| \phi\langle\nabla \phi, \nabla|\omega|\rangle \\
& \leq\left(1+\frac{1}{\varepsilon}\right) \int_{M}|\omega|^{2}|\nabla \phi|^{2}+(1+\varepsilon) \int_{M}|\nabla| \omega||^{2} \phi^{2},
\end{aligned}
$$

where we used Schwarz inequality and Young's inequality for $\varepsilon>0$ in the last inequality. Combining the inequalities (4) and (5), we have

$$
0 \leq\left\{\left(1+\frac{1}{\varepsilon}\right) \frac{1-\delta}{n-1}+1\right\} \int_{M}|\omega|^{2}|\nabla \phi|^{2}
$$




$$
+\left\{(1+\varepsilon) \frac{1-\delta}{n-1}-\frac{1}{n-1}\right\} \int_{M}|\nabla| \omega||^{2} \phi^{2}-\frac{1}{n} \int_{M}|A|^{2}|\omega|^{2} \phi^{2} .
$$

Now fix a point $p \in M$ and consider a geodesic ball $B_{p}(R)$ of radius $R$ centered at $p$. Choose a test function $\phi$ satisfying that $0 \leq \phi \leq 1, \phi \equiv 1$ on $B_{p}(R)$, $\phi \equiv 0$ on $M \backslash B_{p}(2 R)$, and $|\nabla \phi| \leq \frac{1}{R}$.

Letting $R \rightarrow \infty$, then let $\varepsilon \rightarrow 0$, and using the fact that $\int_{M}|\omega|^{2}<\infty$, we finally obtain

$$
\int_{M}|A|^{2}|\omega|^{2}=0
$$

which implies that $A^{2}|\omega|^{2}=0$. Therefore, by (2), we obtain

$$
|\omega| \Delta|\omega| \geq\left.\frac{1}{n-1}|\nabla| \omega\right|^{2}-\frac{(1-\delta) \rho}{2 n-1}|\omega|^{2} .
$$

Combining Lemma 2.1, (6) with the $\mathcal{P}_{\rho}$ property, we obtain

$$
\begin{aligned}
& \left(\frac{1}{n-1}(1-\varepsilon)+1\right) \int_{M} \rho \phi^{2}|\omega|^{2} \\
\leq & \left(\frac{1}{n-1}(1-\varepsilon)+1\right) \int_{M}|\nabla(\phi|\omega|)|^{2} \\
\leq & \frac{1-\delta}{2 n-1} \int_{M} \rho \phi^{2}|\omega|^{2}+\left(\frac{1}{n-1}\left(\frac{1}{\varepsilon}-1\right)+1\right) \int_{M}|\omega|^{2}|\nabla \phi|^{2}
\end{aligned}
$$

for any $\varepsilon>0$ and any compactly supported smooth function $\phi \in \mathcal{C}_{0}^{\infty}(M)$. The above inequality implies

$$
\begin{aligned}
& \left(\frac{n}{n-1}-\frac{1-\delta}{2 n-1}\right) \int_{M} \rho \phi^{2}|\omega|^{2} \\
\leq & \frac{\varepsilon}{n-1} \int_{M} \rho \phi^{2}|\omega|^{2}+\left(\frac{1}{n-1}\left(\frac{1}{\varepsilon}-1\right)+1\right) \int_{M}|\omega|^{2}|\nabla \phi|^{2} .
\end{aligned}
$$

Let

$$
\phi= \begin{cases}1 & \text { on } B(R), \\ 0 & \text { on } M \backslash B(2 R),\end{cases}
$$

such that $|\nabla \phi|^{2} \leq \frac{C}{R^{2}}$ on $B(2 R) \backslash B(R)$. We also choose $\epsilon=R^{\alpha / 2-2}$, by using the growth condition on $\rho$, the above inequality infers

$$
\begin{aligned}
& \left(\frac{n}{n-1}-\frac{1-\delta}{2 n-1}\right) \int_{B(R)} \rho|\omega|^{2} \\
\leq & \frac{R^{-\alpha / 2}}{n-1} \int_{B(2 R)}|\omega|^{2}+\left(\frac{1}{n-1}\left(R^{2-\alpha / 2}-1\right)+1\right) \frac{C}{R^{2}} \int_{B(2 R) \backslash B(R)}|\omega|^{2} .
\end{aligned}
$$

Since $|\omega| \in L^{2}(M)$, we let $R \rightarrow \infty$, finally we conclude that

$$
\int_{M}|\omega|^{2} \leq 0
$$

Hence, $|\omega|=0$. The proof is complete. 
Corollary 2.5. Let $N$ be $(n+1)$-dimensional Riemannian manifold, and $M$ be a complete noncompact stable minimal hypersurface in $N$ with $\lambda_{1}(M)>0$. Assume that

$$
K_{N} \geq-\frac{\lambda_{1}(M)}{(2 n-1)(n-1)}+\delta
$$

for some $\delta>0$, and $K_{N}$ stands for sectional curvature of $N$. Then there are no nontrivial $L^{2}$ harmonic 1 -forms on $M$.

Proof. As a notice in Section 1 , since $\lambda_{1}(M)>0$, we conclude that $M$ satisfies a weighted Poincaré inequality with $\rho \equiv \lambda_{1}(M)$. Obviously, the growth condition is satisfied. By the assumption on sectional curvature of $N$, we see that there is a constant $\delta^{\prime}$ such that

$$
K_{N} \geq-\frac{\left(1-\delta^{\prime}\right) \lambda_{1}(M)}{(2 n-1)(n-1)}
$$

The proof is followed by using Theorem 2.4 .

Corollary 2.6. Let $N$ be $(n+1)$-dimensional Riemannian manifold, and $M$ be a complete noncompact stable minimal hypersurface in $N$ with $\lambda_{1}(M)>0$. Assume that

$$
K_{N} \geq-\frac{\lambda_{1}(M)}{(2 n-1)(n-1)}+\delta
$$

for some $\delta>0$, and $K_{N}$ stands for sectional curvature of $N$. Then $M$ must have only one end.

Proof. The proof is standard, for example, see [2]. We omit the detail.

Acknowledgment. We would like to express our gratitude to Dr. Nguyen Thac Dung for suggesting the problem and many useful discussions on the content of this note.

\section{References}

[1] X. Cheng and D. T. Zhou, Manifolds with weighted Poincaré inequality and uniqueness of minimal hypersurfaces, Comm. Anal. Geom. 17 (2009), no. 1, 139-154.

[2] N. T. Dung and K. Seo, Stable minimal hypersurfaces in a Riemannian manifold with pinched negative sectional curvature, Ann. Global Anal. Geom. 41 (2012), no. 4, 447-460.

[3] K. H. Lam, Results on a weighted Poincaré inequality of complete manifolds, Trans. Amer. Math. Soc. 362 (2010), no. 10, 5043-5062.

[4] P. F. Leung, An estimate on the Ricci curvature of a submanifold and some applications, Proc. Amer. Math. Soc. 114 (1992), no. 4, 1051-1061.

[5] P. Li and J. Wang, Complete manifolds with positive spectrum, J. Differential Geom. 58 (2001), no. 3, 501-534.

[6] X. Wang, On conformally compact Einstein manifolds, Math. Res. Lett. 8 (2001), no. 5-6, 671-688. 
Nguyen Dinh SAng

Department of MATHEMATiCs

Hanoi University of Sciences, (VNU-HUS)

Nguyen Trai street, Thanh Xuan

HANOI, Vietnam

E-mail address: ndsang@gmail.com

Nguyen Thi Thanh

Tran Phu High School for the Gifted

No. 12, Tran Phu street, Ngo Quyen district

Hai Phong City, Vietnam

E-mail address: thanhchuyentp@gmail.com 